\title{
IMAGES OF REAL HYPERSURFACES UNDER HOLOMORPHIC MAPPINGS
}

\author{
M. S. BAOUENDI \& LINDA PREISS ROTHSCHILD
}

\section{Introduction}

In this paper we consider two closely related questions. Given a germ of a real analytic hypersurface $M$ through 0 in $C^{n+1}$ and a germ $H$ at 0 of a holomorphic mapping of $\mathbf{C}^{n+1}$ into itself, under what conditions does there exist some smooth or real analytic hypersurface $M^{\prime}$ in $C^{n+1}$ such that $H(M) \subset M^{\prime}$ ? A complete answer to this question is given for the case where $M$ is essentially finite (see Theorems 1 and 2). Second, given two hypersurfaces $M$ and $M^{\prime}$ in $\mathrm{C}^{n+1}$ and a holomorphic mapping $H$ for which $H(M) \subset M^{\prime}$, what is the structure of $H$ ?

A (germ of a) real analytic hypersurface in $C^{n+1}$ is a set of the form

$$
\left\{Z \in \mathbf{C}^{n+1}: \rho(Z, \bar{Z})=0\right\},
$$

where $\rho$ is a (germ of a) real-valued real analytic function, $\rho(0)=0$, and $d \rho(0) \neq 0$. We may choose holomorphic coordinates $(z, w) \in \mathbf{C}^{n} \times \mathbf{C}$, such that $M$ is given by

$$
\operatorname{Im} w=\phi(z, \bar{z}, s), \quad \text { with } s=\operatorname{Re} w,
$$

where $\phi$ is real analytic and satisfies $\phi(0)=0$ and $d \phi(0)=0$. We may also require that $\phi(z, 0,0) \equiv 0$. With this condition, the $(z, w)$ are called standard coordinates for $M$, and $w$ is called a transversal coordinate. If $H$ is a holomorphic map from $M$ into $M^{\prime}$, we write $H=(f, g)$ if $z^{\prime}=f(z, w)$ and $w^{\prime}=g(z, w)$, where $\left(z^{\prime}, w^{\prime}\right)$ are standard coordinates for $M^{\prime}$. Then $g$ is called a transversal component of $H$, and $(f, g)$ are standard components of $H$.

Recall that a map $H: \mathbf{C}^{n+1} \rightarrow \mathrm{C}^{n+1}$ is finite (at 0$)$ if the ideal $(H(Z)$ ) in the ring of formal power series $\mathrm{C}[[Z]]$ generated by all the components $H_{j}(Z), j=1, \cdots, n+1$, is of finite codimension, i.e., $\operatorname{dim}_{\mathrm{C}} \mathbf{C}[[Z]] /(H(Z))$ $=d$ is finite. We shall call $d$ the multiplicity of $H$ at 0 . In fact, $d$ is

Received December 16, 1990 and, in revised form, July 24, 1991. This research was supported by National Science Foundation Grant DMS-8901268. 
the number of preimages of $H$, counted with multiplicity (see, e.g., [11]). In order to obtain theorems on the nature of mappings, we shall restrict ourselves to the case where $M$ is essentially finite, as introduced in [4]. Recall that if $M$ is given by $(0.2)$ and

$$
\Phi(z, \zeta, 0)=\sum_{\alpha} a_{\alpha}(z) \zeta^{\alpha}
$$

we say that $M$ is essentially finite at 0 if the ideal $\left(a_{\alpha}(z)\right)$ in the ring of formal power series $\mathbf{C}[[z]]$ generated by all the $a_{\alpha}(z)$ is of finite codimension, i.e., $\left.\operatorname{dim}_{\mathbf{C}} \mathbf{C}[z]\right] /\left(a_{\alpha}(z)\right)=m$ is finite. We shall call $m$ the essential type of $M$ at 0 . Note that if $M$ does not contain any germ of a nontrivial complex analytic variety through 0 , then $M$ is essentially finite.

The problem of describing images of complex analytic manifolds and varieties under finite maps is a classical one and well understood (see, e.g., [16], [12], [11], [17]). Less investigation has been devoted to the mixed problem of studying the images of real manifolds under proper holomorphic mappings. Fundamental results have been obtained by D'Angelo [1], [2], concerning the order of contact of complex analytic varieties with real hypersurfaces, as well as the interpretation of these properties in the language of commutative algebra. Interesting questions also have arisen in studying holomorphic extension of Cauchy-Riemann mappings between real hypersurfaces; we refer here to the joint work of the authors with Bell [3] dealing with two complex dimensions, as well as [5]. In [6], the authors proved several properties of holomorphic mappings which take one real hypersurface into another. In particular, we showed that if the first hypersurface is essentially finite, then either a transversal component of the mapping is identically zero, or its first derivative is nonzero and the mapping is finite. These results are essential for the theorems obtained here. Images of hypersurfaces under nonfinite mappings are also studied in this paper.

If $M$ is given by the defining function $\rho$ as in (0.1), we define the germ $\mathscr{M}$ of a complex hypersurface in $\mathrm{C}^{2 n+2}$ by

$$
\mathscr{M}=\left\{(Z, \zeta) \in \mathbf{C}^{2 n+2}: \rho(Z, \zeta)=0\right\},
$$

where $\rho(Z, \zeta)$ is the complex holomorphic function obtained from the real analytic function $\rho$. If $H$ is a holomorphic self-map of $\mathbf{C}^{n+1}$ we associate it to a self-mapping $\mathscr{H}$ of $\mathrm{C}^{2 n+2}$ by

$$
\mathscr{H}(Z, \zeta)=(H(Z), \bar{H}(\zeta)) \text {, }
$$

where $\bar{H}$ is the holomorphic function obtained from $H$ by conjugation of the coefficients in its power series expansion. Note that if $H$ is finite, of multiplicity $d$, then $\mathscr{H}$ is finite with multiplicity $d^{2}$. We note that the 
complexification of a real analytic hypersurface as well as the complexification of a holomorphic mapping given by $(0.3)$ and $(0.4)$ have long been standard in this field since the work of Segre [18], and more recently that of Chern [8], Faran [9], Webster [19], and others.

The main results of this paper may now be stated.

Theorem 1. Let $M$ be a (germ of an) essentially finite real analytic hypersurface in $\mathbf{C}^{n+1}$ through 0 , and let $H$ be a (germ at 0 of a) holomorphic mapping of $\mathbf{C}^{n+1}$ into itself. Then there exists a real analytic hypersurface $M^{\prime}$ such that $H(M) \subset M^{\prime}$ if and only if one of the properties (A) or (B) below is satisfied.

(A) $H\left(\mathbf{C}^{n+1}\right)$ is contained in a (smooth) complex analytic hypersurface of $\mathbf{C}^{n+1}$.

(B) The following three conditions are satisfied:

(i) $H$ is finite;

(ii) $\mathscr{H}^{-1}(\mathscr{H}(\mathscr{M})) \subset \mathscr{M}$; and

(iii) there exists a holomorphic function $J: \mathbf{C}^{n+1} \rightarrow \mathbf{C}, J(0)=0$, such that if $(z, w)$ are standard coordinates for $M$, then $(J \circ H)(z, w)=$ $w K(z, w)$, with $K$ holomorphic and $K(0) \neq 0$.

Moreover, suppose that (A) holds. Then for any real analytic hypersurface $M^{\prime \prime}$ such that $H(M) \subset M^{\prime \prime}$, all of $H\left(\mathbf{C}^{n+1}\right)$ lies in $M^{\prime \prime}$ as a proper subset.

If we are interested in the question of whether $M$ maps into a hypersurface which is assumed only to be smooth rather than real analytic, then we have the following results. We deal first with the criterion that $H$ be a mapping onto a smooth hypersurface.

Theorem 2. Let $M$ be a (germ of an) essentially finite real analytic hypersurface in $\mathbf{C}^{n+1}$ through 0 , and let $H$ be a (germ at 0 of a) holomorphic mapping of $\mathbf{C}^{n+1}$ into itself. Then $H(M)$ is a (smooth) real hypersurface in $\mathbf{C}^{n+1}$ if and only if property (B) of Theorem 1 holds. In addition, if $(\mathrm{B})$ is satisfied, then $H(M)$ is real analytic.

For the last result we need to impose an additional assumption on the smooth target hypersurface $M^{\prime}$. Following D'Angelo [1] we say that a smooth hypersurface $M^{\prime}$ is of $D$-finite type at 0 if there is no nontrivial complex analytic variety through 0 with infinite order of contact with $M^{\prime}$ at 0 . If $M^{\prime}$ is real analytic, $D$-finiteness at 0 is equivalent to the property that there is no nontrivial complex analytic variety through 0 contained in $M^{\prime}$ (see [2] and [14]). In $C^{2}$ the notion of $D$-finite type agrees with the definition of finite type introduced earlier by Kohn [13] using commutators of Cauchy-Riemann vector fields and their conjugates. 
Theorem 3. If $M$ is as in Theorems 1 and 2, and $H$ is a holomorphic self-map of $\mathbf{C}^{n+1}$ such that $H(M) \subset M^{\prime}$, where $M^{\prime}$ is a smooth hypersurface of D-finite type, then either $H$ is finite and $H(M)=M^{\prime}$ with $M^{\prime}$ real analytic, or $H \equiv 0$.

Theorem 3 was essentially proved in [3] in the simpler special case of $n=1$, i.e., $M$ and $M^{\prime}$ hypersurfaces in $\mathrm{C}^{2}$. (See also a generalization of Theorem 3 given by Theorem $3^{\prime}$ in $\S 3$.)

The plan of the present paper is the following. In $\S 1$, we prove Theorem 2 in the case where $M^{\prime}$ is real analytic; examples and remarks are also given. In $\S 2$, we prove a general result which states that if the image of a real analytic manifold under a proper holomorphic mapping is smooth, then it is real analytic. This is needed to complete the proof of Theorem 2 in the case where $M^{\prime}$ is merely smooth. Finally, $\S 3$ is devoted to the proofs of Theorems 1 and 3, followed by further comments and open problems.

The authors wish to thank the referee for a number of useful comments and in particular for suggesting the inclusion of a generalization of Theorem 3.

\section{Mapping a hypersurface onto a hypersurface. Proof of Theorem 2 and remarks}

We shall now prove Theorem 2 . Recall that $\mathscr{M}$ is the complex analytic hypersurface in $C^{2 n+2}$ defined by $(0.3)$ and $\mathscr{H}$ is the holomorphic selfmapping of $\mathrm{C}^{2 n+2}$ defined by (0.4). Assume first that $H(M)=M^{\prime}$ is a real analytic hypersurface in $C^{n+1}$; we shall show that property (B) of Theorem 1 holds. By [6, Theorem 4], if $g$ is a transversal component of $H$, and $w$ a transversal coordinate for $M$, then either $g$ is identically 0 or $\frac{\partial g}{\partial w}(0) \neq 0$ and $H$ is finite. We show first that $g \not \equiv 0$. Indeed, if $g \equiv 0$, then $H(M)$ would be contained in a complex hypersurface which is of real codimension 2 in $\mathbf{C}^{n+1}$, and could not be a real hypersurface of real codimension 1 .

Hence we may assume $H$ is finite and $\frac{\partial g}{\partial w}(0) \neq 0$. The latter condition implies (iii) of (B) since $g(z, w)$ is always of the form $w g_{1}(z, w)$, with $g_{1}(z, w)$ holomorphic. It remains to show (ii). Let $\rho^{\prime}\left(z^{\prime}, w^{\prime}, \bar{z}^{\prime}, \bar{w}^{\prime}\right)=0$ be the defining equation for $M^{\prime}$, with standard coordinates $\left(z^{\prime}, w^{\prime}\right)$ and $\rho^{\prime}$ real analytic. We define a holomorphic function $r$ in $\mathrm{C}^{2 n+2}$ by

$$
\begin{array}{r}
r(z, w, \zeta, \tau)=\rho^{\prime}(f(z, w), g(z, w), \bar{f}(\zeta, \tau), \bar{g}(\zeta, \tau)), \\
z, \zeta \in \mathbf{C}^{n}, w, \tau \in \mathbf{C},
\end{array}
$$


where $f$ and $g$ are standard components of $H$. Since $\rho^{\prime}\left(z^{\prime}, w^{\prime}, 0,0\right)=$ $\alpha w^{\prime}, \alpha(0) \neq 0$, and $\frac{\partial g}{\partial w}(0) \neq 0$, it follows from $(1.1)$ that $\frac{\partial r}{\partial w}(0) \neq 0$.

Let $\mathscr{M}^{\prime}$ be the complex hypersurface in $\mathrm{C}^{2 n+2}$ associated to $M^{\prime}$ given by $(0.3)$ with $\rho$ replaced by $\rho^{\prime}$. The real analyticity of $\rho^{\prime}$ implies that $\mathscr{H}(\mathscr{M}) \subset \mathscr{M}^{\prime}$. We claim that $r$ is a defining function for $M$. Indeed, since $H$ maps $M$ into $M^{\prime}$, it follows from (1.1) that $r(z, w, \bar{z}, \bar{w})$ vanishes on $M$. The claim follows since the differential of $r$ does not vanish. Now to prove (ii) of (B), we observe that if $\left(z_{0}, w_{0}, \zeta_{0}, \tau_{0}\right) \in \mathscr{M}$, then $r\left(z_{0}, w_{0}, \zeta_{0}, \tau_{0}\right)=0$, and hence by $(1.1), r(z, w, \zeta, \tau)=0$ for any $(z, w, \zeta, \tau)$ for which $\mathscr{H}(z, w, \zeta, \tau)=\mathscr{H}\left(z_{0}, w_{0}, \zeta_{0}, \tau_{0}\right)$. This proves the necessity of conditions (i)-(iii).

For the converse, we assume that (i)-(iii) hold. We shall prove that the image $H(M)$ is a real analytic hypersurface in $\mathbf{C}^{n+1}$. For $\left(z^{\prime}, \zeta^{\prime}\right)$ small in $\mathbf{C}^{2 n+2}$, we define $\rho^{\prime}\left(z^{\prime}, \zeta^{\prime}\right)$ as follows. Since $\mathscr{H}$ is a finite map, for each $\left(z^{\prime}, \zeta^{\prime}\right)$ there are $d^{2}$ preimages in $\mathbf{C}^{2 n+2}$, counted with multiplicity, where $d$ is the multiplicity of $H$. We set

$$
\rho^{\prime}\left(Z^{\prime}, \zeta^{\prime}\right)=\sum_{1 \leq j, k \leq d} \rho\left(Z^{j}, \zeta^{k}\right),
$$

where $H\left(Z^{j}\right)=Z^{\prime}, \bar{H}\left(\zeta^{k}\right)=\zeta^{\prime}, 1 \leq j, k \leq d$. Since the right-hand side of (1.2) is a symmetric function of the preimages under $\mathscr{H}$, it follows that $\rho^{\prime}$ is a holomorphic function (see, e.g., [11]).

We claim next that $\rho^{\prime}\left(Z^{\prime}, \bar{Z}^{\prime}\right)$ is real valued. Indeed, the reality of $\rho(Z, \bar{Z})$ implies that for any $(Z, \zeta) \in \mathbf{C}^{n+1}$ we have

$$
\rho(Z, \zeta)=\bar{\rho}(\zeta, Z) \text {. }
$$

We also have

$$
\sum_{1 \leq j, k \leq d} \rho\left(Z^{j}, \zeta^{k}\right)=\sum_{1 \leq j, k \leq d} \rho\left(Z^{j}, \bar{Z}^{k}\right) \text { when } \zeta^{\prime}=\bar{Z}^{\prime},
$$

since $\left\{\bar{Z}^{k}, 1 \leq k \leq d\right\}=\bar{H}^{-1}\left(\bar{Z}^{\prime}\right)$. The reality of $\rho^{\prime}\left(Z^{\prime}, \bar{Z}^{\prime}\right)$ follows by taking the complex conjugate of the right-hand side of (1.4) and using (1.3).

We shall now show that $d \rho^{\prime}(0) \neq 0$. After making holomorphic changes of variables and using (iii), we may assume that

$$
H=\left(H_{1}, \cdots, H_{n+1}\right), \quad \text { with } H_{n+1}(z, w)=w,
$$

and from now on we shall write $H=\left(f_{1}, \cdots, f_{n}, g\right)$. We denote by $(z, w, \zeta, \tau)$ the new holomorphic coordinates in the source space, and 
by $\left(z^{\prime}, w^{\prime}, \zeta^{\prime}, \tau^{\prime}\right)$ those of the target space. Then (1.2) becomes

$$
\rho^{\prime}\left(z^{\prime}, w^{\prime}, \zeta^{\prime}, \tau^{\prime}\right)=\sum_{1 \leq j, k \leq d} \rho\left(z^{j}, w^{\prime}, \zeta^{k}, \tau^{\prime}\right),
$$

with $f\left(z^{j}, w^{\prime}\right)=z^{\prime}, \bar{f}\left(\zeta^{j}, \tau^{\prime}\right)=\zeta^{\prime}$. To compute $\left(\partial \rho^{\prime} / \partial w^{\prime}\right)(0)$, it suffices to take $z^{\prime}=\zeta^{\prime}=0$ and $\tau^{\prime}=0$ in (1.6). Since

$$
\begin{aligned}
\rho(z, w, 0,0)= & a w+\left(\sum_{|\alpha| \geq 1} a_{\alpha 1} z^{\alpha}\right) w \\
& +\sum_{|\alpha| \geq 0, p \geq 2} a_{\alpha p} z^{\alpha} w^{p}, \quad a \neq 0,
\end{aligned}
$$

with $a$ and $a_{\alpha p}$ constants, by (1.6) we have

$$
\rho^{\prime}\left(0, w^{\prime}, 0,0\right)=d^{2} a w^{\prime}+\left(\sum_{|\alpha| \geq 1} a_{\alpha 1} k_{\alpha}\left(w^{\prime}\right)\right) w^{\prime}+\mathscr{O}\left(\left|w^{\prime}\right|^{2}\right),
$$

with $k_{\alpha}\left(w^{\prime}\right)=d \sum_{1 \leq j \leq d}\left(z^{j}\right)^{\alpha}$, where the $z^{j}$ are the $d$ solutions of $f\left(z^{j}, w^{\prime}\right)=0$.

Note that the $k_{\alpha}$ are holomorphic functions, since they are symmetric functions of the preimages of $\left(0, w^{\prime}\right)$ under the map $H$. We also have $k_{\alpha}(0)=0$. Hence, by (1.8), $\left(\partial \rho^{\prime} / \partial w^{\prime}\right)(0)=d^{2} a \neq 0$.

Now let $M^{\prime}$ be the hypersurface defined by $\rho^{\prime}\left(z^{\prime}, w^{\prime}, \vec{z}^{\prime}, \bar{w}^{\prime}\right)=0$, which is real analytic by construction. To prove that $H(M) \subset M^{\prime}$ we must use (1.2) together with condition (ii), which until this point has not been used. Indeed, if $(Z, \zeta) \in \mathscr{M}$, i.e., $\rho(Z, \zeta)=0$, then by (1.2) $\rho^{\prime}(H(Z), \bar{H}(\zeta))=\sum_{j, k} \rho\left(Z_{j}, \zeta_{k}\right)$, where $H\left(Z_{j}\right)=H(Z)$ and $\bar{H}\left(\zeta_{k}\right)=\bar{H}(\zeta)$. Then (ii) implies that each term in the last sum is zero, i.e., $\mathscr{H}(Z, \zeta) \in \mathscr{M}^{\prime}$, which means that $H(M) \subset M^{\prime}$.

It remains to prove that $H(M)=M^{\prime}$. This is a consequence of the following.

Lemma 1.9. Let $H$ be a finite map in $C^{n+1}$, and $M$ and $M^{\prime}$ real analytic hypersurfaces with $H(M) \subset M^{\prime}$. Then $H(M)=M^{\prime}$.

Proof. The conclusion follows from the study of multiplicities of finite mappings in [6] but can also be easily proved directly as follows. Let $(z, w)$ and $\left(z^{\prime}, w^{\prime}\right)$ be standard coordinates for $M$ and $M^{\prime}$ respectively, and let $Z^{\prime}=\left(z^{\prime}, w^{\prime}\right) \in M^{\prime}$, i.e., $\rho^{\prime}\left(Z^{\prime}, \bar{Z}^{\prime}\right)=0$. Since any finite map is surjective, there exists $Z_{0}=\left(z_{0}, w_{0}\right) \in \mathbf{C}^{n+1}$ such that $H\left(Z_{0}\right)=Z^{\prime}$. We claim that $Z_{0} \in M$. For this, as in (1.1), let $r(Z, \bar{Z})=\rho^{\prime}(H(Z), \bar{H}(\bar{Z}))$. Since $\rho^{\prime}\left(z^{\prime}, w^{\prime}, 0,0\right)=\alpha w^{\prime}, \alpha(0) \neq 0$, and $\frac{\partial g}{\partial w}(0) \neq 0$, it follows that 
$\frac{\partial r}{\partial w}(0) \neq 0$, i.e., $r$ is a defining function for $M$. The lemma follows by taking $Z=Z_{0}$. q.e.d.

We have now proved that (i)-(iii) of property (B) of Theorem 1 are necessary and sufficient for $H(M)$ to be a real analytic hypersurface. Theorem 4 of $\S 2$ below shows that $H(M)$ need not be assumed to be real analytic; indeed, it is shown more generally that if the image of a smooth real analytic manifold under a finite holomorphic map is smooth, then it must also be real analytic. This will complete the proof of Theorem 2 .

Remark 1.10. The smoothness assumption in Theorem 2 cannot be weakened by assuming instead that $M^{\prime}$ is of class $C^{p}$ for some finite $p$. Indeed, let $M=\left\{(z, w) \in \mathbf{C}^{2}: \operatorname{Im} w=|z|^{2 k}\right\}$ with $k$ odd, $M^{\prime}=$ $\left\{\left(z^{\prime}, w^{\prime}\right) \in \mathbf{C}^{2}: \operatorname{Im} w^{\prime}=|z|^{k}\right\}$, and let $H=\left(z^{2}, w\right)$. It is easy to check that $H(M)=M^{\prime}$, and that $M^{\prime}$ is not smooth, but is of class $C^{p}$ if $k$ is large enough.

Remark 1.11. The above example also shows that condition (ii) of property (B) cannot be replaced in Theorem 2 by the weaker condition

(ii' $\left.{ }^{\prime}\right) H^{-1}(H(M)) \subset M$.

Indeed, for $K=1$, for instance, (i) and (iii) are obviously satisfied, and $H^{-1}\left(z^{2}, s+i|z|^{2}\right)=\left( \pm z, s+i|z|^{2}\right) \in M$. Hence (ii') is satisfied, but $H(M)$ is not a smooth hypersurface.

\section{Image of a real analytic manifold under a finite map}

We prove here the following result.

Theorem 4. Let $H$ be a (germ at the origin of a) finite holomorphic mapping from $\mathbf{C}^{N}$ to itself with $H(0)=0$. Let $M$ be a (germ at the origin of a) real analytic submanifold of $\mathbf{C}^{N}$. Assume that $H(M)=M^{\prime}$ is a smooth submanifold of $\mathbf{C}^{N}$. Then $M^{\prime}$ is real analytic.

The theorem will follow from a seemingly more restrictive result:

Proposition 2.1. Let $J$ be a (germ at the origin of a) finite holomorphic mapping from $\mathbf{C}^{n}$ to itself with $J(0)=0$ and $J$ real, i.e., $J\left(\mathbf{R}^{n}\right) \subset \mathbf{R}^{n}$. Let $M$ be a (germ at the origin of a) real analytic submanifold of $\mathbf{R}^{n}$. If $J(M)=M^{\prime}$ is a smooth submanifold of $\mathbf{R}^{n}$, then $M^{\prime}$ is real analytic.

Proof. Since $M \subset \mathbf{R}^{n}$ and is real analytic, after a local holomorphic change in $\mathbf{C}^{n}$, we may assume that $M=\left\{x_{1}, \cdots, x_{n-k}, 0, \cdots, 0\right)$, with the usual notation $z=x+i y$. Let $W=\left\{z \in \mathbf{C}^{n}:|z|<\varepsilon\right\}$ and $U=J^{-1}(W)$. If $\varepsilon$ is sufficiently small, then the map $J: U \rightarrow W$ is a proper surjective map (see, e.g., [11], [17]). Let $A$ be the subvariety of $U$ given by $U \cap\left\{z_{n-k+1}=\cdots=z_{n}=0\right\}$ and $B=J(A) \subset W$. Note that 
$M \cap\left\{x \in \mathbf{R}^{n}:|x|<\varepsilon\right\}=A \cap \mathbf{R}^{n}$. By Remmert's Proper Mapping Theorem [16], $B$ is a subvariety of $W$. Hence $B \cap \mathbf{R}^{n}$ is a real analytic set containing $M^{\prime}$.

We wish to apply the following theorem due to Malgrange [15]: Let $X_{0}$ be the germ of a real analytic set in $\mathbf{R}^{n}$ through 0 containing a germ $M_{0}$ of a smooth manifold. If $\operatorname{dim} X_{0}=\operatorname{dim} M_{0}$, then $M_{0}$ is real analytic.

We take here $X_{0}=B \cap \mathbf{R}^{n}$ and $M_{0}=J(M)=M^{\prime}$. In order to apply Malgrange's theorem, we need only check that

$$
\operatorname{dim}_{R} M^{\prime}=\operatorname{dim}_{\mathbf{R}} B \cap \mathbf{R}^{n} .
$$

First, by considering points of maximal rank on $M$, we see that $\operatorname{dim}_{\mathrm{R}} M^{\prime}$ $=\operatorname{dim}_{\mathrm{R}} M=n-k$. Indeed, a holomorphic map at points of maximal rank is either injective or not proper. Similarly, we have $\operatorname{dim}_{\mathrm{C}} B=n-k$. For $M^{\prime}$ we note first that by the inclusion $M^{\prime} \subset B \cap \mathbf{R}^{n}$, we have $\operatorname{dim} M^{\prime} \leq$ $\operatorname{dim}_{\mathbf{R}} B \cap \mathbf{R}^{n}$. Finally, we observe that $\operatorname{dim}_{\mathbf{R}} B \cap \mathbf{R}^{n} \leq \operatorname{dim}_{\mathbf{C}} B$. This proves (2.2), which shows that Malgrange's theorem can be applied to yield the proof of Proposition 2.1. q.e.d.

We shall now prove Theorem 4 . We first identify $M$ as a submanifold of $\mathbf{R}^{n}, n=2 N$, by the usual identification of $\mathbf{C}^{N}$ with $\mathbf{R}^{2 N} ; M$ is then a real analytic submanifold of $\mathbf{R}^{n}$. The map $H$ can then be identified with a real analytic mapping $J: \mathbf{R}^{n} \rightarrow \mathbf{R}^{n}$ given by

$$
J(x, y)=\left(\frac{1}{2}[H(x+i y)+\bar{H}(x-i y)], \frac{1}{2 i}[H(x+i y)-\bar{H}(x-i y)]\right) .
$$

Since $J(M)=M^{\prime}$ can be regarded as a smooth submanifold of $\mathbf{R}^{n}$, we may apply Proposition 2.1 if we can show that $J$ extends to $\mathbf{C}^{n}$ as a finite holomorphic map. To do this, we first complexify $J$ by taking $x$, $y$ in $C^{2 N}$ in (2.3). To prove that the resulting holomorphic map, which we again denote by $J$, is finite, it suffices to prove that the components of $J$ have no common zeros near the origin, other than the origin itself. The latter follows immediately from the finiteness of the map $H$ and (2.3). This proves Theorem 4 .

Remark 2.4. Note that without the assumption that $M^{\prime}$ is smooth, one cannot even conclude that $J(M)$ is a real analytic set, as is shown by the example of Remark 1.10 .

\section{Holomorphic mappings into a hypersurface. Proof of Theorems 1 and 3}

In this section we shall prove Theorems 1 and 3 . We recall the following result from [6]: Let $M$ and $H$ be as in the assumption of Theorem 1, 
with $M^{\prime}$ a real analytic hypersurface for which $H(M) \subset M^{\prime}$. We write $H=(f, g)$, where $g$ is a transversal component for $H$. If $w$ is a transversal coordinate for $M$, then either $g \equiv 0$ or $\frac{\partial g}{\partial w}(0) \neq 0$ and $H$ is finite.

Assume now that $H(M) \subset M^{\prime}$ as in Theorem 1. If $g \not \equiv 0$, then by the above $H$ is finite and hence, by Lemma $1.9, H(M)=M^{\prime}$. Therefore, we are done by Theorem 2 . In order to prove the theorem we may assume $g \equiv 0$. It is clear that property (A) of Theorem 1 holds since then $H\left(\mathbf{C}^{n+1}\right)$ is contained in the complex hyperplane $w^{\prime}=0$. To complete the proof of Theorem 1 , we must show that if $g \equiv 0$, then $H\left(\mathbf{C}^{n+1}\right)$ is a proper subset of $M^{\prime}$.

We denote by $\mathrm{rk}_{\mathrm{C}} H^{\prime}(Z)$ the rank of the holomorphic map $H$ at $Z$, regarded as a map from $\mathbf{C}^{n+1}$ into itself. Similarly, for $Z \in M$ we denote by $\mathrm{rk}_{M} H^{\prime}(Z)$ the rank of $H$ at $Z$ considered as a map from $M$ into $\mathbf{C}^{n+1}$ (identified with $\mathbf{R}^{2 n+2}$ ).

The following will be used in the proof of Theorem 1.

Proposition 3.1. If $H$ is a holomorphic mapping from $\mathbf{C}^{n+1}$ into $\mathbf{C}^{p}$, and $M$ is a smooth hypersurface in $\mathbf{C}^{n+1}$, then, for every $Z \in M$,

$$
\mathrm{rk}_{\mathrm{C}} H^{\prime}(Z)=\left[\frac{1}{2}\left(\mathrm{rk}_{M} H^{\prime}(Z)+1\right)\right],
$$

where $[k]$ denotes the greatest integer less than or equal to $k$.

Proof. Without loss of generality we may assume $Z=0$. We choose standard coordinates $(z, w)$ for $M$. We may assume that $M$ is given by (0.2), and use $(z, \bar{z}, s)$ as coordinates on $M$, with $z \in \mathbf{C}^{n}, s \in \mathbf{R}$, and $s=\operatorname{Re} w$. By identifying $\mathbf{C}^{p}$ with $\mathbf{R}^{2 p}$ by the correspondence $\mathbf{C}^{p} \ni \zeta \mapsto$ $(\zeta, \bar{\zeta})$, the map $H: M \rightarrow \mathrm{C}^{p}$ is then given by

$$
(z, \bar{z}, s) \mapsto K(z, \bar{z}, s)=(H(z, s+i \phi(z, \bar{z}, s)), \bar{H} \bar{z}, s-i \phi(z, \bar{z}, s)))
$$

Since $d \phi(0)=0$, the rank of $K$ at 0 is the rank of the matrix

$$
\operatorname{Jac}(K)=\left(\begin{array}{cc}
H_{z}(0) & 0 \\
H_{w}(0) & \bar{H}_{\bar{w}}(0) \\
0 & \bar{H}_{\bar{z}}(0)
\end{array}\right),
$$

where $H_{z}(0)$ is the $p \times n$ Jacobian matrix of $H$ with respect to $z$, and $H_{w}(0)$ is the $p \times 1$ derivative of $H$ with respect to $w$.

We now consider the two cases corresponding to whether $\operatorname{rk}_{M} H^{\prime}(0)$ is even or odd. In the first case, $\operatorname{rk}(\operatorname{Jac}(K))=2 \operatorname{rk}\left(H_{z}\right)$, and the row $H_{w}$ 
is in the span of the rows of $H_{z}$. Thus $\operatorname{rk}_{\mathrm{C}} H^{\prime}(0)=\operatorname{rk}\left(H_{z}\right)(0)$. In the second case, $\operatorname{rk}(\operatorname{Jac}(K))=2 \operatorname{rk}\left(H_{z}\right)+1$ and $\operatorname{rk}_{\mathrm{C}} H^{\prime}(0)=\operatorname{rk}\left(H_{z}\right)(0)+1$. Hence equality (3.2) is proved.

Corollary 3.5. Let $H$ be as in Proposition 3.1. If the restriction of $H$ to $M$ has maximal rank at $m_{0} \in M$, then $H$ has maximal rank at $M_{0}$ as a complex holomorphic mapping.

Proof. The proof is immediate from (3.2), since $\mathrm{rk}_{\mathrm{C}} H^{\prime}(Z)$ is determined by $\operatorname{rk}_{M} H^{\prime}(Z)$. Note that the converse is false. For example, let $M$ be the Lewy hypersurface in $\mathbf{C}^{2}$ given by $\left\{(z, w): \operatorname{Im} w=|z|^{2}\right\}$, and $H(z, w)=(w, 0)$. Then $\mathrm{rk}_{\mathrm{C}} H^{\prime}(0)=1$, which is maximal, but $\mathrm{rk}_{M} H^{\prime}(0)=1$, while $\mathrm{rk}_{M} H^{\prime}(Z)=2$ for $Z \neq 0$. q.e.d.

We may now return to the proof of Theorem 1. As noted above, we may assume $g \equiv 0$. Let $r$ be the largest integer such that for every neighborhood $U$ of 0 in $M$ there is a point $Z \in U$ with $\mathrm{rk}_{M} H^{\prime}(Z)=r$. Let $m_{0} \in M$ be any point sufficiently close to 0 at which $\mathrm{rk}_{M} H^{\prime}\left(m_{0}\right)=r$.

Suppose first that $r$ is even, i.e., $r=2 p$. By Corollary $3.5, \mathrm{rk}_{\mathrm{C}} H^{\prime}\left(m_{0}\right)$ is also maximal, and is equal to $p$ by (3.2). It follows that $H(M)=$ $H\left(\mathbf{C}^{n+1}\right)=V$ near $m_{0}$, where $V$ is a smooth complex holomorphic submanifold of dimension $p$. We claim that $H\left(\mathbf{C}^{n+1}\right) \subset M^{\prime}$ near 0 also. Indeed, let $\rho^{\prime}$ be a defining function for $M^{\prime}$. Since $\rho^{\prime}(H(Z), \bar{H}(\bar{Z}))$ is real analytic in a neighborhood of 0 and vanishes identically for $Z$ in a complex neighborhood of $m_{0}$, we conclude that it vanishes identically in a complex neighborhood of 0 in $\mathbf{C}^{n+1}$ also, which proves the claim. By dimension, it is clear that the inclusion must be proper.

We shall show that $r$ is in fact always even. For this, suppose by contradiction that $r$ is odd, i.e., $r=2 p+1$. By (3.2), $\operatorname{rk}_{\mathrm{C}} H^{\prime}\left(m_{0}\right)=$ $p+1$, and $M_{0}$ is a point of maximal rank. Note that $p<n$ since $g \equiv 0$. Hence near $M_{0}, H(M)$ is a hypersurface $M^{\prime \prime}$ in $H\left(\mathbf{C}^{n+1}\right)=V$, where $V$ is a $(p+1)$-dimensional smooth complex submanifold of $\mathbf{C}^{n}$. After holomorphic changes of coordinates, we may make $V=\mathbf{C}^{p+1}$, and may assume that $M_{0}=0$ and that $H(Z)=\left(Z_{1}, \cdots, Z_{p+1}\right)$ near 0 . Let $\rho^{\prime \prime}$ be a defining function for $M^{\prime \prime}$. We conclude that $\rho^{\prime \prime}\left(Z_{1}, \cdots\right.$, $\left.Z_{p+1}, \bar{Z}_{1}, \cdots, \bar{Z}_{p+1}\right)$ is also a defining function for $M$. Since $p<n, \rho^{\prime \prime}$ is independent of the variables $Z_{p+2}, \cdots, Z_{n+1}$. This implies that $M$ is not essentially finite at $m_{0}$. Since essential finiteness is an open condition [6], and $m_{0}$ may be chosen arbitrarily close to 0 , this contradicts the assumption that $M$ is essentially finite at 0 . Therefore $r$ cannot be an odd number. 
We conclude from the above that $r$ is necessarily even and $H\left(\mathbf{C}^{n+1}\right) \subset$ $M^{\prime}$. Also, since $\operatorname{dim} M^{\prime}=2 n+1$ is odd, $H(M) \neq M^{\prime}$. Hence the proof of Theorem 1 is complete.

Remark 3.6. If property (A) of Theorem 1 holds so that $H(M) \subset M^{\prime}$ for some real analytic hypersurface $M^{\prime}$, then it is still possible that $H(M)$ is a proper subset of $H\left(\mathrm{C}^{n+1}\right)$ (which is a proper subset of $M^{\prime}$ by Theorem 1). For instance, suppose that $M$ is the Lewy hypersurface in $\mathbf{C}^{2}$ given by $\operatorname{Im} w=|z|^{2}$ and $H=(w, 0)$. Here $M^{\prime}$ is the flat hypersurface $\operatorname{Im} w^{\prime}=0$. Then $H\left(\mathbf{C}^{2}\right)=\mathbf{C} \times 0$, while $H(M)=\mathbf{C}^{+} \times 0$, where $\mathbf{C}^{+}$is the upper half plane of $C$.

The proof of Theorem 3 is obtained as a modification of the proof of Theorem 1. For this, we recall some terminology from [6]. If $M^{\prime}$ is a smooth hypersurface in $\mathbf{C}^{n+1}$ through 0 and $j$ is a positive integer, then holomorphic coordinates $\left(z^{\prime}, w^{\prime}\right)$ are called standard of order $j$ if $M^{\prime}$ is given by

$$
\operatorname{Im} w^{\prime}=\psi\left(z^{\prime}, \vec{z}^{\prime}, s^{\prime}\right), \quad \text { with } s^{\prime}=\operatorname{Re} w^{\prime},
$$

with $\psi$ smooth and $\psi\left(z^{\prime}, 0,0\right) \equiv 0$ up to order $j+1$; in this case $w^{\prime}$ is called a transversal coordinate of order $j$. By a formal transversal coordinate (of order $\infty$ ) we shall mean a holomorphic formal power series such that for every finite $j$ there is a transversal coordinate of order $j$ whose Taylor expansion agrees with that of the formal series up to order $j$. Similarly, we may define formal standard components or standard components of order $j$, and formal transversal components or transversal components of order $j$ for a holomorphic mapping $H: M \rightarrow M^{\prime}$.

We shall need the following lemma.

Lemma 3.8. Let $M$ and $M^{\prime}$ be smooth hypersurfaces in $\mathbf{C}^{n+1}$ and $H: \mathbf{C}^{n+1} \rightarrow \mathbf{C}^{n+1}$ holomorphic and mapping $M$ into $M^{\prime}$. If a formal transversal component of $H$ vanishes identically, then $\operatorname{det} \operatorname{Jac}(H) \equiv 0$.

Proof. Assume by contradiction that $\operatorname{det} \operatorname{Jac}(H) \not \equiv 0$, so that there exists a finite integer $k$ with $\operatorname{det} \operatorname{Jac}(H)$ vanishing of order exactly $k$ at 0 . After a holomorphic transformation in the target space, we can write $H=(f, g)$, with $g$ a transversal component of order $k+1$. Then by the hypothesis, $\operatorname{det} \operatorname{Jac}(H)$ in these coordinates vanishes of order at least $k+1$. Since the order of vanishing of the determinant is an invariant under holomorphic change of coordinates, we reach a contradiction, and the lemma is thus proved. q.e.d.

In order to reduce to the case where a formal transversal component of $H$ vanishes identically, we need the following strengthening of Lemma 1.9 . 
Lemma 3.9. Let $H$ be a finite map in $\mathbf{C}^{n+1}$, and $M$ and $M^{\prime}$ smooth hypersurfaces with $H(M) \subset M^{\prime}$ ). Then $H(M)=M^{\prime}$.

The proof of Lemma 3.9 is almost identical to that of Lemma 1.9, in which both $M$ and $M^{\prime}$ are assumed to be real analytic, except that one takes standard coordinates $(z, w)$ and $\left(z^{\prime}, w^{\prime}\right)$ of order one, and takes $g$ to be a transversal component of order one.

We may now complete the proof of Theorem 3. If a formal transversal component of $H$ does not vanish identically, then, since $M$ is essentially finite, by [6, Theorem 4], $H$ is finite. By Lemma 3.9, we conclude that $H(M)=M^{\prime}$, and we are in the situation of Theorem 2, so there is nothing more to prove. Therefore we may assume that a formal transversal component $g$ of $H$ vanishes identically, and hence by Lemma 3.8, $\operatorname{det} \operatorname{Jac}(H) \equiv 0$. We must use the $D$-finiteness of $M^{\prime}$ to show that $H \equiv 0$. As in the proof of Theorem 1, we let $r$ be the maximal rank of the restriction of $H$ to $M$. Let $m_{0} \in M$ be any point sufficiently close to 0 at which $\mathrm{rk}_{M} H^{\prime}\left(m_{0}\right)=r$. Since $\operatorname{det} \operatorname{Jac}(H) \equiv 0,(3.2)$ implies that $r<2 n+1$. The essential finiteness of $M$ thus yields that $r$ must be even by the same argument as used in Theorem 1. We claim that $r$ must be 0 . Assume that $r=2 p$. By Corollary 3.5, $\mathrm{rk}_{\mathrm{C}} H^{\prime}\left(m_{0}\right)$ is also maximal, and is equal to $p$ by (3.2). It follows $H(M)=H\left(\mathbf{C}^{n+1}\right)=V \subset M^{\prime}$ near $m_{0}$, where $V$ is a smooth complex holomorphic submanifold of dimension $p$. Since $m_{0}$ is arbitrarily close to 0 , the germ of the variety $h\left(\mathbf{C}^{n+1}\right)$ at 0 has infinite order of contact with $M^{\prime}$. Indeed, if $\rho^{\prime}$ is a smooth defining function for $M^{\prime}$, we have $\rho^{\prime}(H(Z), \overline{H(Z))} \equiv 0$ in a complex neighborhood of $m_{0}$ and hence $\rho^{\prime}(H(Z), \overline{H(Z)})$ vanishes of infinite order at the origin in $C^{n+1}$. This would contradict the D-finiteness of $M^{\prime}$ unless $r=0$. The claim is now proved and the proof of Theorem 3 is complete. q.e.d.

A smooth hypersurface $M^{\prime}$ is of $q$ D-finite type at 0 , where $q$ is a positive integer, if there is no complex analytic variety of dimension greater than or equal to $q$ through 0 with infinite order of contact with $M^{\prime}$ at 0 . Hence $M^{\prime}$ is of D-finite type if and only if it is of $1 \mathrm{D}$-finite type. The proof of Theorem 3 above can be easily modified to yield the following generalization.

Theorem $3^{\prime}$. If $M$ is as in Theorems 1 and 2, and $H$ is a holomorphic self-map of $\mathrm{C}^{n+1}$ such that $H(M) \subset M^{\prime}$, where $M^{\prime}$ is a smooth hypersurface of $q$ D-finite type, $q$ being a positive integer, then either $H$ is finite and $H(M)=M^{\prime}$ with $M^{\prime}$ real analytic, or the generic rank of $H$ is less than or equal to $q-1$ in a neighborhood of 0 and the variety $H\left(\mathbf{C}^{n+1}\right)$ has infinite order of contact with $M^{\prime}$.

Remark 3.10. Suppose that $H(M) \subset M^{\prime}$, with $M$ real analytic and 
essentially finite and with $M^{\prime}$ smooth but $H$ not finite. If $M^{\prime}$ is either smooth of D-finite type or real analytic, then it follows from Theorems 1 and 3 that $H\left(\mathbf{C}^{n+1}\right) \subset M^{\prime}$. This inclusion need not hold in general, as shown by the following example. As in Remark 3.6, let $M$ be the Lewy hypersurface and $H=(w, 0)$. Let $M^{\prime}$ be the hypersurface in $\mathbf{C}^{2}$ given by $\operatorname{Im} w^{\prime}=\phi\left(\operatorname{Im} z^{\prime}\right)$, where $\phi(t)$ is a smooth function of one real variable vanishing for $t>0$ and nonvanishing in any neighborhood of 0 . Then $H(M) \subset M^{\prime}$, and $H\left(\mathbf{C}^{2}\right)=\mathrm{C} \times 0$ is not a subset of $M^{\prime}$ but is only tangent of infinite order to $M^{\prime}$ at 0 .

Remark 3.11. Suppose that $H(M) \subset M^{\prime}$, with $M$ real analytic and essentially finite and with $M^{\prime}$ smooth but $H$ not finite. Here again if $M^{\prime}$ is real analytic, it follows from Theorem 1 that a transversal component $g$ of $H$ vanishes identically, and hence $H\left(\mathbf{C}^{n+1}\right)$ is contained in a smooth holomorphic hypersurface $V$ of $\mathbf{C}^{n+1}$. We do not know whether or not the same conclusion holds if the real analyticity of $M^{\prime}$ is dropped, although in this case a formal transversal component necessarily vanishes identically. The existence of such $V$ is equivalent to a nondegenerate holomorphic relation between the components of $H$. When $M^{\prime}$ is smooth, the vanishing of a formal transversal component of $H$ yields a nondegenerate formal holomorphic relation between the components of $H$. In general, such a relation between convergent power series does not imply the existence of a convergent holomorphic relation, as was shown by an example of Gabrielov [10]. We thank T. T. Moh for showing us this reference.

Remark 3.12. If $M$ is not assumed to be essentially finite in Theorem 2 , then property (B) of Theorem 1 need not hold. In particular, a holomorphic mapping $H$ of a hypersurface $M$ onto a hypersurface $M^{\prime}$ can be finite but with vanishing differential at 0 . For this, take $M=M^{\prime}$ in $\mathbf{C}^{2}$ given by $\operatorname{Im} w=0$ and $H(z, w)=\left(z^{2}, w^{3}\right)$. Also, one can have a mapping $H$ which is onto in the sense of germs but not finite. For this, take $M=M^{\prime}$ in $\mathbf{C}^{3}$ given by $\operatorname{Im} w=0$, and $H(z, w)=\left(f_{1}(z), f_{2}(z), w\right)$, where $f(z)=\left(f_{1}(z), f_{2}(z)\right)$ is any surjective mapping of $\mathbf{C}^{2}$ onto itself, in the sense of germs, but which is not finite. For instance, we can take $f_{1}(z)=z_{1} z_{2}^{2}$ and $f_{2}(z)=z_{2}\left(z_{2}-z_{1}\right)$.

\section{References}

[1] J. P. D'Angelo, Real hypersurfaces, orders of contact, and applications, Ann. of Math. (2) 115 (1982) 615-637.

[2] __ Finite type and the intersection of real and complex subvarieties, Proc. Sympos. Pure Math. (E. Bedford, J. P. D' Angelo, R. E. Greene, and S. G. Krantz, eds.), Vol. 52, Part 3, Amer. Math. Soc., Providence, RI, 1991, 103-118. 
[3] M. S. Baouendi, S. R. Bell \& L. P. Rothschild, Mappings of three-dimensional CR manifolds and their holomorphic extension, Duke Math. J. 56 (1988) 503-530.

[4] M. S. Baouendi, H. Jacobowitz \& F. Treves, On the analyticity of $C R$ mappings, Ann. of Math. (2) 122 (1985) 365-400.

[5] M. S. Baouendi \& L. P. Rothschild, Germs of CR maps between real analytic hypersurfaces, Invent. Math. 93 (1988) 481-500.

[6] - Geometric properties of smooth and holomorphic mappings between hypersurfaces in complex space, J. Differential Geometry 31 (1990) 473-499.

[7] __ Holomorphic mappings of real analytic hypersurfaces, Proc. Sympos. Pure Math., (E. Bedford, J. P. D' Angelo, R. E. Greene, and S. G. Krantz, eds.), Vol. 52, Part 3, Amer. Math. Soc., Providence, RI, 1991, 15-26.

[8] S. S. Chern, On the projective structure of a real hypersurface in $C^{n}$, Math. Scand. 36 (1975) 74-82.

[9] J. J. Faran, Segre families and real hypersurfaces, Invent. Math. 60 (1980) 135-172.

[10] A. M. Gabrielov, Formal relations between analytic functions, Funktsional. Anal. i Prilozhen. 5 (1971) 64-65.

[11] P. Griffiths \& J. Harris, Principles of algebraic geometry, Wiley Interscience, New York, 1978.

[12] R. Gunning \& H. Rossi, Analytic functions of several complex variables, Prentice-Hall, Englewood Cliffs, NJ, 1965.

[13] J. J. Kohn, Boundary behavior of $\bar{\partial}$ on weakly pseudoconvex manifolds of dimension two, J. Differential Geometry 6 (1972) 523-542.

[14] L. Lempert, On the boundary behavior of holomorphic mappings, Contributions to Several Complex Variables in Honour of Wilhelm Stoll, Vieweg and Son, Braunschweig 1986, 193-215.

[15] B. Malgrange, Ideals of differentiable functions, Tata Institute of Fundamental Research, Oxford University Press, Bombay, 1966.

[16] R. Remmert, Holomorphe und meromorphe Abbildungen komplexer Räume, Math. Ann. 133 (1957) 328-370.

[17] W. Rudin, Function theory on the unit ball in $\mathbf{C}^{n}$, Springer, Berlin, 1980.

[18] B. Segre, Intorno al problema di Poincaré della rappresentazione pseudo-confrom, Rend. Accad. Lincei 13 (1931) 676-683.

[19] S. M. Webster, On the mapping problem for algebraic real hypersurfaces, Invent. Math. 43 (1977) 53-68. 